\title{
Study of anomalous mobility of polar molecular solutions by means of the site-site memory equation formalism
}

\author{
A. E. Kobryn, ${ }^{1}$ T. Yamaguchi, ${ }^{2}$ and F. Hirata, ${ }^{1, *}$ \\ ${ }^{1}$ Department of Theoretical Study, Institute for Molecular Science, Myodaiji, Okazaki, Aichi 444-8585, Japan \\ ${ }^{2}$ Department of Molecular Design and Engineering, Graduate School of Engineering, \\ Nagoya University, Chikusa, Nagoya, Aichi 464-8603, Japan
}

(Dated: November 11, 2004)

\begin{abstract}
In this work, the memory equation approach is applied for theoretical study of dynamics of polar molecular liquids described by the interaction site model. The study includes the temperature-density(pressure) dependence of the translational diffusion coefficients $D$ and orientational relaxation times $\tau$ for infinitely dilute solutions of acetonitrile and methanol in water, and methanol in acetonitrile. Calculations are performed over the range of temperatures and densities employing the SPC/E model for water and optimized site-site potentials for acetonitrile and methanol. Despite an approximate character of the model potentials and closure relation used, the theory is able to reproduce qualitatively all main features of temperature and density dependences of $D$ and $\tau$ observed in computer and real experiments. In particular, anomalous behavior, i.e. the increase in mobility with density(pressure), is obtained for $D$ and $\tau$ of methanol in water, while acetonitrile in water or methanol in acetonitrile do not show deviations from the usual. The observed enhancement in the molecular mobility is interpreted in accordance with the concept by Yamaguchi et al. [J. Chem. Phys. 119 (2003) 1021], i.e. in terms of two competing origins of friction, which interplay with each other as density increases: the collisional and dielectric frictions that have tendency, respectively, to strengthen and weaken with increasing density.
\end{abstract}

Keywords: Translational Diffusion; Rotational Relaxation; Memory Equation; Mode-Coupling; RISM

\section{INTRODUCTION}

The enhancement of the molecular mobility of polar molecules in dense dipolar liquids under pressure, called anomalous molecular mobility, has recently attracted considerable attention in solution chemistry experiments and simulations 1, 2, 3, 4, 5, 6, 7, 8, 9]. Conventional continuum-based theories of liquid matter collapsed when applied for the description of anomalous molecular behavior, so that to make theoretical justification of the observed processes is topical from the point of view stationed on the statistical mechanical approach [10]. In order to clarify the microscopic structure of water, which is a typical representative of a liquid with anomalous behavior, and its mobility, it has been studied recently by Yamaguchi et al. using the memory equation approach for molecular liquids described by the interaction site model [1]. The authors concluded, that anomalous pressure dependence of dynamic properties of water can be explained by the strong interatomic Coulomb interaction correlated with density fluctuations. In particular, when the number-density fluctuation in the low wavenumber region decreases with increasing pressure, the Coulomb coupling between the low wave-number chargedensity fluctuation and hydrodynamic dielectric mode is reduced. In the case of another polar liquids, like acetonitrile, the effect of Coulomb interaction can be blocked by even more substantial repulsive interaction, which makes

\footnotetext{
*The author to whom correspondence should be sent; Electronic address: hirata@ims.ac.jp URL: daisy.ims.ac.jp
}

it dissimilar with water. Phrasing it differently - anomalous molecular mobility strongly depends on the molecular geometry and dominant type of interatomic interaction, and more studies is required for better understanding of its nature. In this paper we pay attention to the study of anomalous pressure behavior of polar binary molecular solutions by considering such popular models as methanol in water, acetonitrile in water and methanol in acetonitrile. In particular, we are interested in the density(pressure) dependence of translational diffusion coefficients and reorientation relaxation times of solutes. The rest of the paper is organized as follows. In section II we write down equations of motion for site-site intermediate scattering functions, and give relations for translational diffusion coefficient and reorientation correlation time in terms of these functions. Equilibrium properties of the system are obtain using DRISM, and memory kernels are estimated by the mode-coupling approximation. Set up for systems under consideration and details of numerical procedures are described in section III Obtained results are discussed in section IV and conclusions are given in section $\nabla$

\section{THEORY}

For the description of time-dependent properties of the system, we will follow the formalism by Hirata and Chong 12], which is the unification of the theory of dynamical processes in simple liquids based on the Mori approach 13. 14] and reference interaction site model for molecular liquids [15, 16, 17]. In their formalism, parameters of shortened description of the system are partial num- 
ber densities and longitudinal current densities. Then, of practical interest are elements of the matrix of the site-site intermediate scattering functions $\mathbf{F}(k ; t)$ and the matrix of their self-parts $\mathbf{F}_{\mathrm{s}}(k ; t)$ defined by

$$
\begin{aligned}
& F^{\alpha \gamma}(k ; t)=\frac{1}{N}\left\langle\rho^{\alpha, *}(\mathbf{k} ; 0) \rho^{\gamma}(\mathbf{k} ; t)\right\rangle, \\
& F_{\mathrm{s}}^{\alpha \gamma}(k ; t)=\frac{1}{N}\left\langle\rho^{\alpha, *}(\mathbf{k} ; 0) \rho^{\gamma}(\mathbf{k} ; t)\right\rangle_{\mathrm{s}},
\end{aligned}
$$

where $N$ is the total number of particles, $\rho^{\alpha}(\mathbf{k} ; t)$ is the site $\alpha$ number density in reciprocal space, $\mathbf{k}$ is the wavevector, $k=|\mathbf{k}|, t$ is time, $*$ means complex conjugation, angular brackets denote appropriate statistical average (e.g., canonical Gibbs ensemble average), and suffix "s" stands for "self" and means correlations between two sites of a same molecule. In the limit of infinite dilution, which is considered here, the solution density is essentially determined by the solvent. Consecutively, one has two types of memory equations for the solvent subsystem and one for the solute subsystem (indicated by the superscript "u") which are:

$$
\begin{gathered}
\ddot{\mathbf{F}}(k ; t)=-\left\langle\boldsymbol{\omega}_{k}^{2}\right\rangle \mathbf{F}(k ; t)-\int_{0}^{t} \mathrm{~d} \tau \mathbf{K}(k ; \tau) \dot{\mathbf{F}}(k ; t-\tau), \\
\ddot{\mathbf{F}}_{\mathrm{s}}(k ; t)=-\left\langle\boldsymbol{\omega}_{k}^{2}\right\rangle_{\mathrm{s}} \mathbf{F}_{\mathrm{s}}(k ; t)-\int_{0}^{t} \mathrm{~d} \tau \mathbf{K}_{\mathrm{s}}(k ; \tau) \dot{\mathbf{F}}_{\mathrm{s}}(k ; t-\tau), \\
\ddot{\mathbf{F}}_{\mathrm{s}}^{\mathrm{u}}(k ; t)=-\left\langle\boldsymbol{\omega}_{k}^{2}\right\rangle_{\mathrm{s}}^{\mathrm{u}} \mathbf{F}_{\mathrm{s}}^{\mathrm{u}}(k ; t)-\int_{0}^{t} \mathrm{~d} \tau \mathbf{K}_{\mathrm{s}}^{\mathrm{u}}(k ; \tau) \dot{\mathbf{F}}_{\mathrm{s}}^{\mathrm{u}}(k ; t-\tau) .
\end{gathered}
$$

In these equations, dot over the quantity means its time derivative; the memory function matrices, denoted as $\mathbf{K}(k ; t), \mathbf{K}_{\mathrm{s}}(k ; t)$ and $\mathbf{K}_{\mathrm{s}}^{\mathrm{u}}(k ; t)$, describe the friction on the motion of interaction sites; quantities $\left\langle\boldsymbol{\omega}_{k}^{2}\right\rangle,\left\langle\boldsymbol{\omega}_{k}^{2}\right\rangle_{\mathrm{s}}$ and $\left\langle\boldsymbol{\omega}_{k}^{2}\right\rangle_{\mathrm{s}}^{\mathrm{u}}$ are normalized second order frequency matrices given by

$$
\begin{aligned}
\left\langle\boldsymbol{\omega}_{k}^{2}\right\rangle & =k^{2} \mathbf{J}(k) \mathbf{S}^{-1}(k), \\
\left\langle\boldsymbol{\omega}_{k}^{2}\right\rangle_{\mathrm{s}} & =k^{2} \mathbf{J}(k) \mathbf{S}_{\mathrm{s}}^{-1}(k), \\
\left\langle\boldsymbol{\omega}_{k}^{2}\right\rangle_{\mathrm{s}}^{\mathrm{u}} & =k^{2} \mathbf{J}^{\mathrm{u}}(k) \mathbf{S}_{\mathrm{s}}^{\mathrm{u},-1}(k),
\end{aligned}
$$

where $\mathbf{S}(k) \equiv \mathbf{F}(k ; t=0), \mathbf{S}_{\mathrm{s}}(k) \equiv \mathbf{F}_{\mathrm{s}}(k ; t=0)$ and $\mathbf{S}_{\mathrm{s}}^{\mathrm{u}}(k) \equiv \mathbf{F}_{\mathrm{s}}^{\mathrm{u}}(k ; t=0)$ are matrices of static site-site structure factors and their self parts, respectively, while $\mathbf{J}(k)$ is the matrix of static longitudinal site-current correlation functions

$$
\lfloor\mathbf{J}(k)\rfloor^{\alpha \gamma}=\frac{1}{N} \sum_{i, j}\left\langle v_{i, z}^{\alpha} v_{j, z}^{\gamma} \mathrm{e}^{-i \mathbf{k} \cdot\left(\mathbf{r}_{i}^{\alpha}-\mathbf{r}_{j}^{\gamma}\right)}\right\rangle,
$$

where subscripts $i, j$ refer to molecules, $\mathbf{r}_{i}^{\alpha} \equiv \mathbf{r}_{i}^{\alpha}(0)$ is the initial position of site $\alpha$, and $v_{i, z}^{\alpha} \equiv v_{i, z}^{\alpha}(0)$ is longitudinal component of the initial velocity of site $\alpha$. The analytical expression of $\mathbf{J}(k)$ for arbitrary shape of the molecule has been given recently by Yamaguchi et al. [18]. Definition for $\mathbf{J}^{\mathrm{u}}(k)$ is similar to the one given by equation (4) with the difference that summation runs over the solute molecule only. Initial values of intermediate scattering functions, that we need to solve memory equations (2), can be obtained using RISM theory [15, 16, 17]. That theory predicts static structure of molecular fluids via the calculation of their site-site pair correlation functions. In this work, however, in order to have a better description for the dielectric susceptibility of solvent we employ the formalism of DRISM that uses experimental value of dielectric constant [19, 20].

Quantities of our interest in this paper are solute's translational diffusion coefficient $D$ and rank-1 reorientation correlation time $\tau$. Following the derivation procedure presented in [21], the translational diffusion coefficient $D$ is obtained as

$$
\begin{aligned}
D & =\frac{1}{3} \int_{0}^{\infty} \mathrm{d} t Z^{\alpha \gamma}(t) \\
& =-\lim _{t \rightarrow \infty} \int_{0}^{t} \mathrm{~d} \tau \lim _{k \rightarrow 0} \frac{1}{k^{2}}\left\lfloor\ddot{\mathbf{F}}_{\mathrm{s}}^{\mathrm{u}}(k ; \tau)\right\rfloor^{\alpha \gamma},
\end{aligned}
$$

where $Z^{\alpha \gamma}(t)$ is the site-site velocity autocorrelation function with sites $\alpha$ and $\gamma$ belonging to the same molecule. The rank-1 reorientation correlation function $C_{\boldsymbol{\mu}}(t)$ is defined by

$$
C_{\boldsymbol{\mu}}(t)=\frac{\sum_{i}\left\langle\boldsymbol{\mu}_{i}(0) \boldsymbol{\mu}_{i}(t)\right\rangle}{\sum_{j}\left\langle\left|\boldsymbol{\mu}_{j}\right|^{2}\right\rangle}
$$

where $\boldsymbol{\mu}_{i}(t)$ is a vector fixed on the molecule $i$. In our case it is the dipole moment and therefore can be described by the linear combination of site coordinates as $\boldsymbol{\mu}_{i}(t)=\sum_{\alpha} z_{\alpha} \mathbf{r}^{\alpha}(t)$ with $z_{\alpha}$ being site partial charges. Putting that into equation (6) and using properties of time-correlation functions 22] one arrives at

$$
\begin{aligned}
C_{\boldsymbol{\mu}}(t) & =\frac{\sum_{i} \sum_{\alpha \gamma} z_{\alpha} z_{\gamma}\left\langle\mathbf{r}_{i}^{\alpha}(0) \mathbf{r}_{i}^{\gamma}(t)\right\rangle}{\sum_{j}\left\langle\left|\boldsymbol{\mu}_{j}\right|^{2}\right\rangle} \\
\ddot{C}_{\boldsymbol{\mu}}(t) & =-\frac{N \sum_{\alpha \gamma} z_{\alpha} z_{\gamma} Z^{\alpha \gamma}(t)}{\sum_{j}\left\langle\left|\boldsymbol{\mu}_{j}\right|^{2}\right\rangle}
\end{aligned}
$$

Hence, the time development of both $Z^{\alpha \gamma}(t)$ and $C_{\boldsymbol{\mu}}(t)$ is governed by the memory equation for the self-part of the site-site intermediate scattering function $\mathbf{F}_{\mathrm{s}}^{\mathrm{u}}(k ; t)$. The rank-1 reorientation relaxation time is defined as [23]

$$
\tau=\int_{0}^{\infty} \mathrm{d} t C_{\boldsymbol{\mu}}(t)
$$

Since the dynamics we are interested in corresponds to the long-time limit, memory kernels for memory equations can be constructed using the mode-coupling approximation [23]. In works by Chong et al. 24, 25] the conventional mode-coupling theory has been extended to the case of molecular liquids based on the interaction-site model. It has been shown, however, that the proposed 
expressions for memory functions underestimate friction in orientational motions 26]. In our study we use the recipe by Yamaguchi and Hirata [26], who suggested a modified expression that includes the interaxial coupling.

\section{DETAILS OF THE MODEL SETUP AND NUMERICAL PROCEDURES}

We performed calculations for acetonitrile in water, methanol in water and methanol in acetonitrile, all in the case of infinite dilution. As for the structure and the intermolecular potential of water we employed a model of the extended simple point charge (SPC/E) 27]. We also put the Lennard-Jones (LJ) core on the hydrogen atoms in order to avoid the undesired divergence of the solution of the DRISM integral equation. The LJ parameters of the hydrogen atom, the depth of the well and the diameter, were chosen to be $0.046 \mathrm{kcal} / \mathrm{mol}$ and $0.7 \AA$, respectively.

In acetonitrile and methanol the methyl group was considered to be a single interaction site located on the methyl carbon atom. So that all chemical compounds consisted of three sites which interact through the pair potential [28, 29]

$$
\phi\left(r_{i j}^{\alpha \gamma}\right)=4 \epsilon_{\alpha \gamma}\left[\left(\frac{\sigma_{\alpha \gamma}}{r_{i j}^{\alpha \gamma}}\right)^{12}-\left(\frac{\sigma_{\alpha \gamma}}{r_{i j}^{\alpha \gamma}}\right)^{6}\right]+\frac{z_{\alpha} z_{\gamma}}{r_{i j}^{\alpha \gamma}},
$$

i.e., LJ plus Coulomb. Here $r_{i j}^{\alpha \gamma}=\left|\mathbf{r}_{i}^{\alpha}-\mathbf{r}_{j}^{\gamma}\right|$; parameters $\epsilon_{\alpha \gamma}$ and $\sigma_{\alpha \gamma}$ are LJ well-depths and LJ diameters defined as $\epsilon_{\alpha \gamma}=\sqrt{\epsilon_{\alpha} \epsilon_{\gamma}}$ and $\sigma_{\alpha \gamma}=\left(\sigma_{\alpha}+\sigma_{\gamma}\right) / 2$, respectively, with $\sigma_{\alpha}$ being the LJ diameter of a single site. Point charges for acetonitrile were chosen to reproduce electrostatic potential obtained in $a b$ initio calculations [28].

In calculations for acetonitrile or methanol in water temperature of the system was varied from 258.15 to $373.15 \mathrm{~K}$, and density of water from 0.9 to $1.2 \mathrm{~g} / \mathrm{cm}^{3}$; for the case of methanol in acetonitrile temperature of the system was varied from 293.15 to $323.15 \mathrm{~K}$, and density of acetonitrile from 0.6726 to $0.815 \mathrm{~g} / \mathrm{cm}^{3}$. Connection of the water parameters with thermodynamic pressure can be established, e.g., using the multi-parametric equation of state [30] (except for the metastable regions where reliable data is lacking).

Temperature/density dependent dielectric constant $\varepsilon$ for water used in numerical calculations has been evaluated as a physical solution of an empirical nonlinear equation [31]:

$$
\varepsilon-\frac{1}{2}\left(1+\frac{1}{\varepsilon}\right)=\frac{1}{v}\left(17+\frac{9.32 \cdot 10^{4}\left(1+\frac{153}{v \cdot T^{1 / 4}}\right)}{(1-3 / v)^{2} T}\right),
$$

where $v$ is a molar volume in units of $\mathrm{cm}^{3} / \mathrm{mol}$, and $T$ is thermodynamic temperature in $\mathrm{K}$. This equation has been used also in such temperature/density points where no experimental values exist. For density and dielectric constant of acetonitrile at different temperatures we used experimental values indicated in Table \

TABLE I: Density and dielectric constant for acetonitrile as functions of temperature: experimental data used in our computation. Temperature is in $\mathrm{K}$, and density is in $\mathrm{g} / \mathrm{cm}^{3}$.

\begin{tabular}{cccccccc}
\hline \hline$T$ & 293.13 & 295.05 & 298.15 & 303.15 & 308.15 & 313.15 & 323.15 \\
\hline$\rho$ & $.782^{\mathrm{a}}$ & - & $.7762^{\mathrm{b}}$ & $.7712^{\mathrm{a}}$ & $.7652^{\mathrm{b}}$ & $.7603^{\mathrm{a}}$ & $.7492^{\mathrm{a}}$ \\
$\varepsilon$ & $38.8^{\mathrm{c}}$ & $37.5^{\mathrm{d}}$ & $36.69^{\mathrm{e}}$ & $35.93^{\mathrm{e}}$ & & & $33.5^{\mathrm{f}}$ \\
\hline \hline
\end{tabular}

a 32$\left.\left.\left.],{ }^{\mathrm{b}} 33\right],{ }^{\mathrm{c}} 34\right],{ }^{\mathrm{d}} 35\right],{ }^{\mathrm{e}}$ 36], ${ }^{\mathrm{f}}$ Extrapolated

In the numerical part of this study we calculated first the site-site static structure factor by solving the DRISM equation using the intermolecular interaction, molecular shape, temperature and density. In order to improve the convergence of the DRISM calculation, we used the method of the modified direct inversion in an iterative space (MDIIS) proposed by Kovalenko et al. 37]. The value of the parameter of DRISM theory $a$ has been set to $0.1 \AA^{2}$. From the static site-site structure factor, we calculated the site-site intermediate scattering function using the site-site memory equation with the mode-coupling approximation for the memory kernels. The memory equation has been time-integrated numerically. Timedevelopment of correlation functions in the $k \rightarrow 0$ limit was treated separately by the analytical limiting procedure of theoretical expressions. In the numerical procedure, the reciprocal space was linearly discretized as $k=\left(n+\frac{1}{2}\right) \Delta k$, where $n$ is an integer from 0 to $N_{k}-1$. Values of $\Delta k$ and $N_{k}$ are $0.061 \AA^{-1}$ and $2^{9}=512$, respectively. The choice for $N_{k}$ as the power of two came as the requirement of the subroutine for the fast Fourier transform, which has been used in DRISM / MDIIS. The diffusion coefficient $D$ was calculated from the asymptotic slope of the time dependence of the mean square displacement, and the orientational relaxation time $\tau$ was obtained by time-integration of the rotational autocorrelation function.

\section{RESULTS AND DISCUSSIONS}

\section{A. Structural properties}

When applied to the liquid state, external pressure induces changes both in its structure and dynamics of molecules. The anomalous behavior of the molecular mobility with pressure, i.e. dynamics, has been assigned to the hydrogen bonding properties of the system, which in its turn can be related to structural properties. The idea is that the hydrogen bond or hydrogen bonding network are distorted upon compression, so that the molecule has less hydrogen bonds at higher pressure, which makes its translational and rotational motion looser. On the other hand, extensive compression results in significant grow of repulsive forces between molecules. In fact, the liq- 

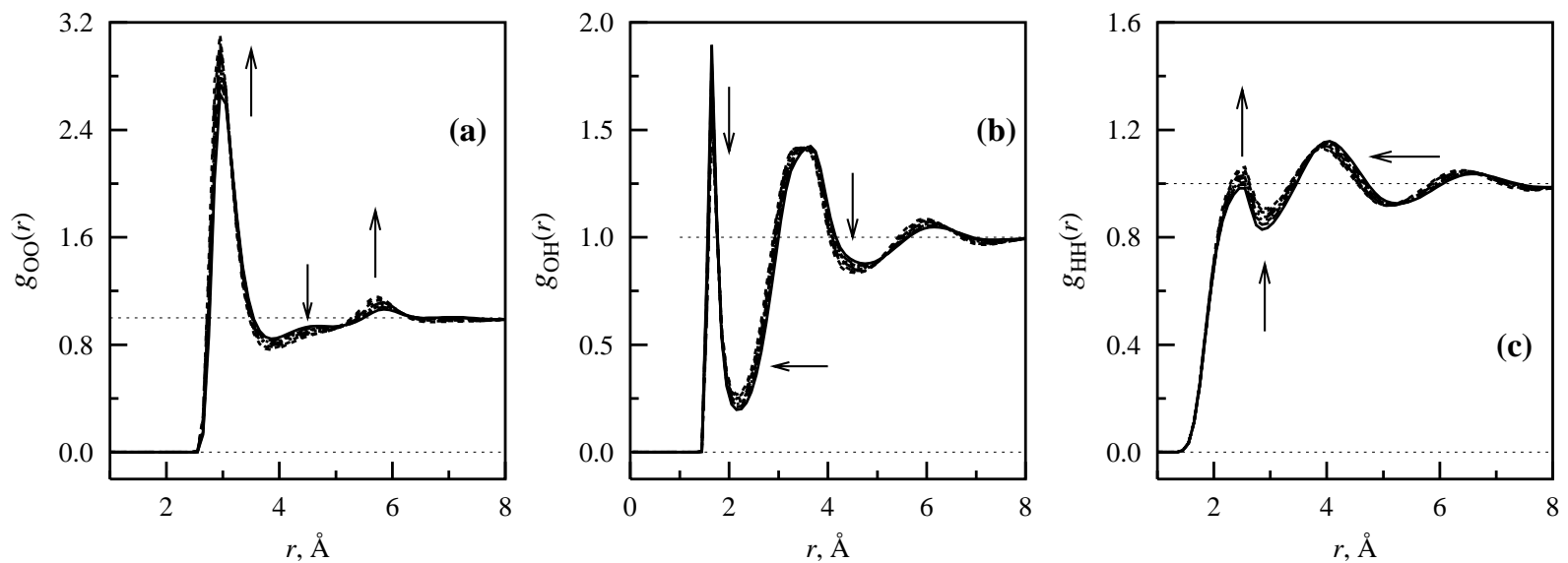

FIG. 1: Site-site radial distribution functions of neat water at $T=273.15 \mathrm{~K}$ and set of densities from 0.9 to $1.125 \mathrm{~g} / \mathrm{cm}^{3}$, obtained by the DRISM/HNC integral equation theory. Arrows show directions of alternations due to an increase in pressure.
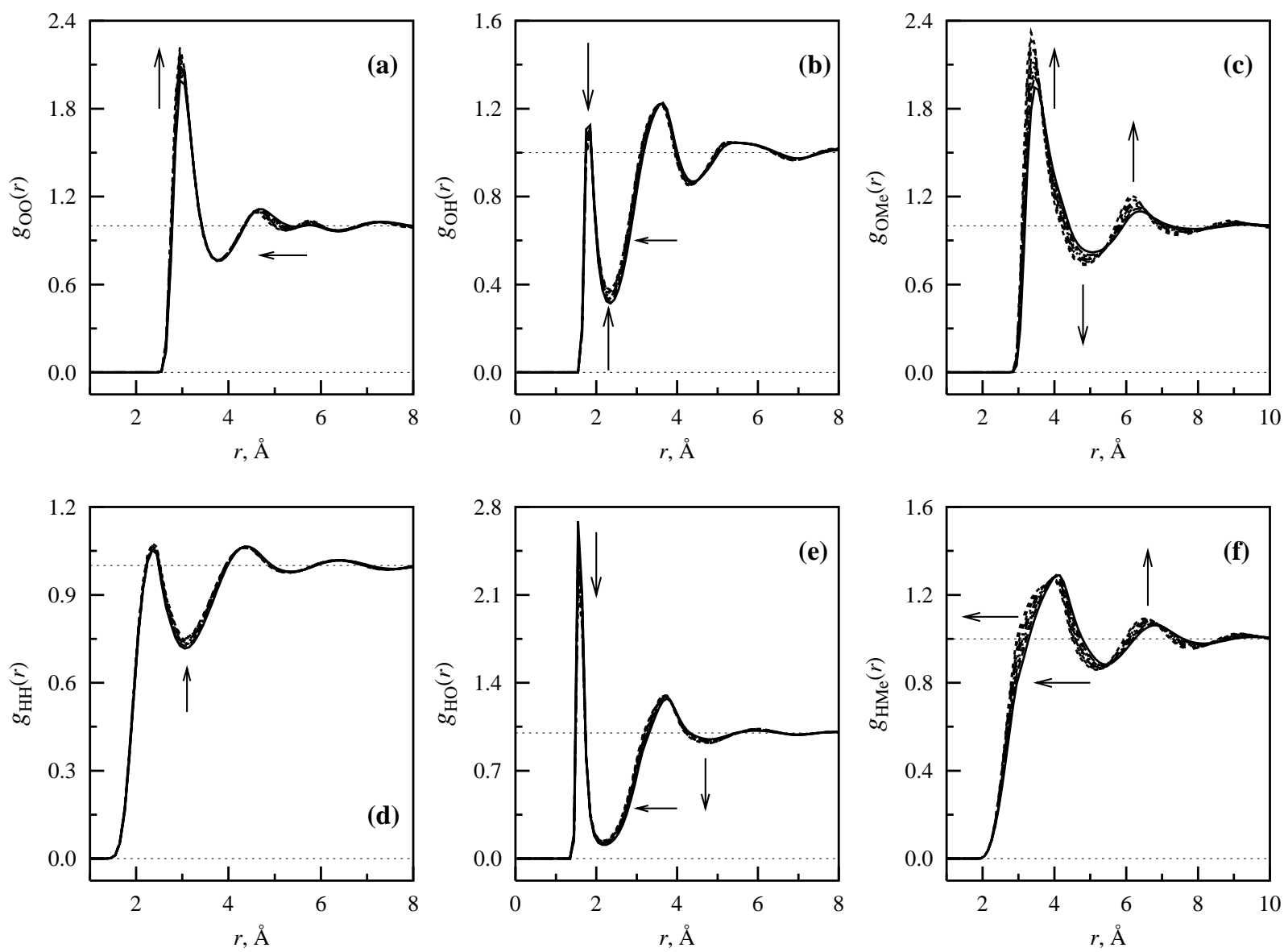

FIG. 2: Water-methanol site-site radial distribution functions at $T=273.15 \mathrm{~K}$ and set of densities from 0.9 to $1.125 \mathrm{~g} / \mathrm{cm}^{3}$ for water, obtained by the DRISM/HNC integral equation theory. In the notations used, first site always belongs to water, and second site always belongs to methanol. Arrows show directions of alternations due to an increase in pressure.

uid structure is determined essentially by these two competing factors: hydrogen bonding and repulsive interaction, which interfere each other through the intramolec- ular constraints or molecular geometry. There was much attention to this topic in scientific literature and we shall not repeat results of these studies here. Instead of that 

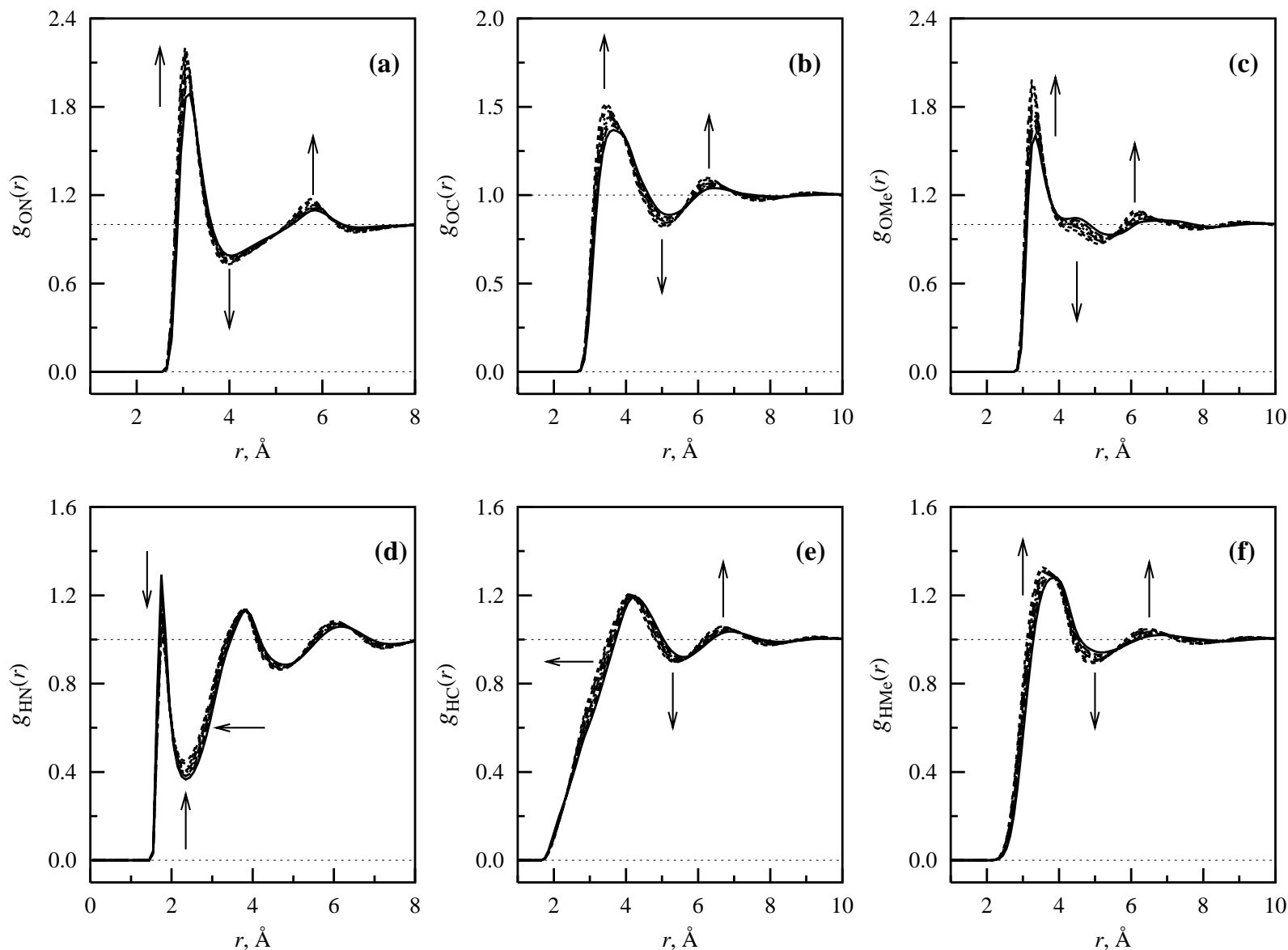

FIG. 3: Water-acetonitrile site-site radial distribution functions at $T=273.15 \mathrm{~K}$ and set of densities from 0.9 to $1.125 \mathrm{~g} / \mathrm{cm}^{3}$ for water, obtained by the DRISM/HNC integral equation theory. In the notations used, first site always belongs to water, and second site always belongs to acetonitrile. Arrows show directions of alternations due to an increase in pressure.

we can focus our analysis on some properties related to the subject. Figs. 11-4] show site-site RDF's for neat water, water-methanol, water-acetonitrile and acetonitrilemethanol infinite dilution solutions, respectively, calculated by the DRISM/NHC theory for set of densities at constant temperature. Despite the comparatively big number of site-site combinations, there are some common features in the behavior of each individual site-site RDF, that can be categorized as follows. First, one sees that there are RDF's with the first peak either remained almost unchanged, or enhanced with the increase of density(pressure). Second, there are first peaks that are located at positions much shorter, than other peaks, and that all of them subside with density(pressure) a lot. The former case is a reflection of packing effect. It is because of the result of pressure pushes the next nearest neighbor toward the central molecule and makes the first coordination shell to be outlined more clearly, which is also confirmed by the drift of majority of peak locations into direction of shorter separations. The effect of pressure also distorts the preferable mutual orientation of molecules, which does not favor hydrogen bonds. The latter case in given previously peak categorization is an example of such state of things. To be more specific, first peaks in Figs. 1(b), 2(b) and 2(e), 3(d), and 4(b) all are evidences of hydrogen bonds in the system, and all of them subside with the increase of density. There is, however, a difference in their nature. For methanol in water the hydrogen bonding is realized in two ways: once when the proton acceptor is solvent, i.e. water, Fig. 2(b); and once when the proton acceptor is the solute, i.e. methanol, Fig. 2(e). In the first case the peak amplitude is much lower, than in the second case, indicating that the first type of hydrogen bonding may be somewhat weaker. Nevertheless, due to the fact that both solvent and solute can evident as both proton donor and acceptor, they are able to make either hydrogen bond network, as in the case of neat water, or hydrogen bond zigzag chains, as in the case of pure methanol or water-methanol mixture. For acetonitrile in water the hydrogen bonding is realized only in one way: when the nitrogen site of acetonitrile serves as the proton acceptor, Fig. 3(d). The peak amplitude of that hydrogen bond is also lower compared to neat water, Fig. 1(b), or water-methanol, Fig. 2(e), but general behavior 

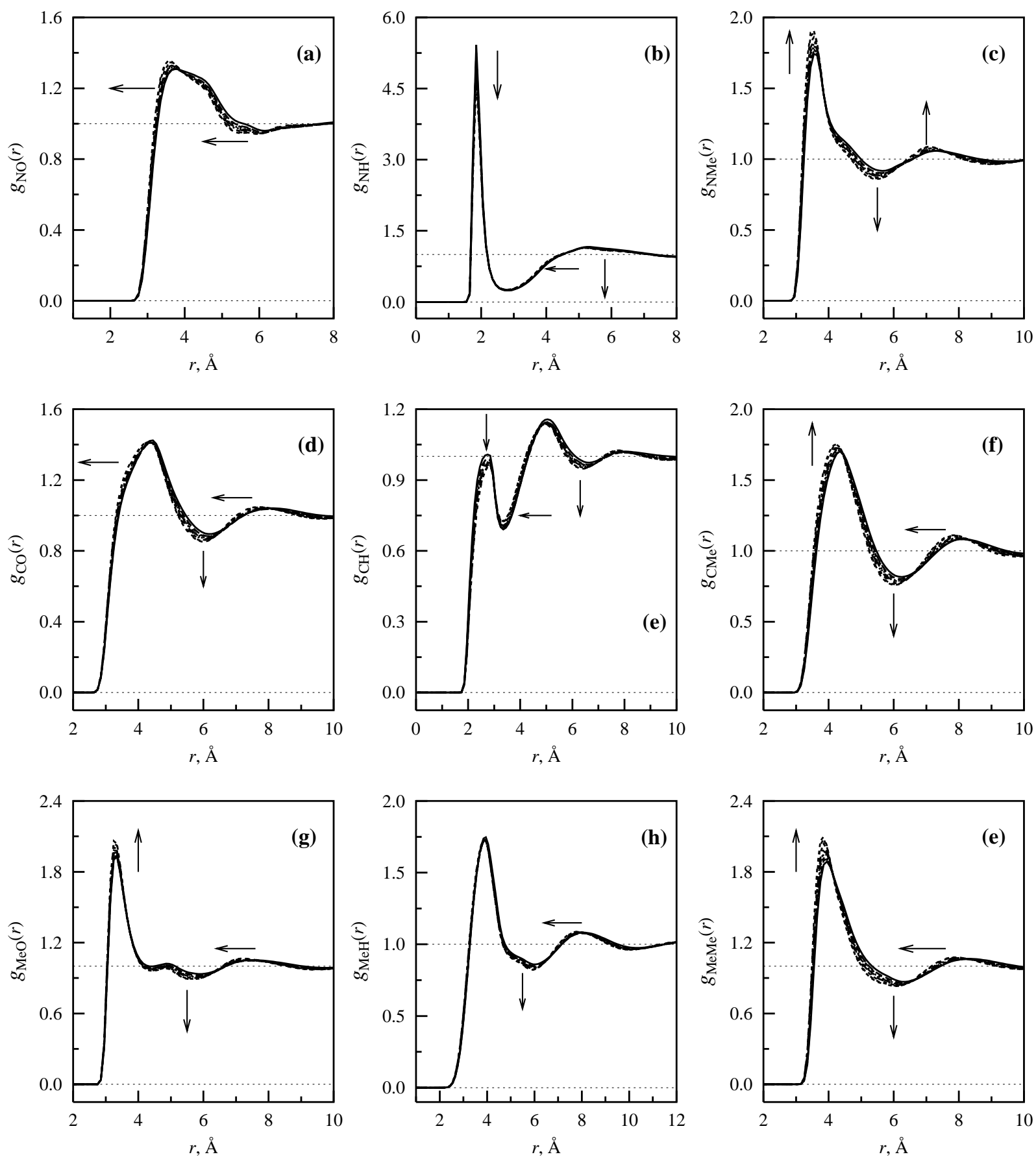

FIG. 4: Acetonitrile-methanol site-site radial distribution functions at $T=293.15 \mathrm{~K}$ and set of densities from 0.6726 to 0.815 $\mathrm{g} / \mathrm{cm}^{3}$ for acetonitrile, obtained by the DRISM/HNC theory. In the notations used, first site always belongs to acetonitrile, and second site always belongs to methanol. Arrows show directions of alternations due to an increase in pressure.

of RDF with density(pressure) is essentially the same. The lack of ability for acetonitrile to serve as the proton acceptor and donor has far reaching consequences and results in impossibility to create a hydrogen bond network neither in pure substance or between water an acetonitrile molecules in their mixture. Thus, the solution of acetonitrile in water represents situation when hydrogen bonds are realized between both types of molecules, but hydrogen bond network is possible to establish between water molecules exclusively. Finally, for methanol in acetonitrile the only hydrogen bond available in the system is between the nitrogen site of the solvent and hydrogen site of the solute, Fig. 世(b). Any type of the hydrogen bond network does not appear in such solution at all. 


\section{B. Dynamical properties}

Fig. 5 shows the density(pressure) dependence of the normalized translational diffusion coefficient $D / D_{0}$ and normalized reorientation relaxation time $\tau / \tau_{0}$ for investigated solutes at various temperatures. Here $D_{0}$ and $\tau_{0}$ are translational diffusion coefficient and reorientation relaxation time, respectively, of the solute at the ambient density for the solvent, which is $\rho=0.997047$ $\mathrm{g} / \mathrm{cm}^{3}$ for water and $\rho=0.782 \mathrm{~g} / \mathrm{cm}^{3}$ for acetonitrile. In particular, Fig. 5(a) shows that for sufficiently low temperature the diffusion coefficient of methanol in water first increases with density (pressure), and then smoothly changes its behavior to the normal one, i.e. decreases with density(pressure). Relatively flat maximum is observed for the density slightly smaller than that at the ambient condition. At higher temperatures maximum is not observed. Similar features can be mentioned about behavior of the diffusion coefficient of acetonitrile in water, Fig. 5(b). But in this case the increase of the diffusion coefficient at the lowest temperature is very small and can be monitored only by comparing numbers for neighboring density points. The cubic spline curve, which connects these points, makes this very flat maximum observed visually. Behavior of the diffusion coefficient at higher temperatures does not deviate from the normal one, as in the case of methanol in water. Experimental measurements of diffusion coefficients for acetonitrile in water and acetonitrile- $\mathrm{D}_{3}\left(\mathrm{CD}_{3} \mathrm{CN}\right)$ in water, both at $T=303 \mathrm{~K}$, reported by Nakahara et al. [2] testify the same tendency in their density(pressure) behavior as obtained from the theory. Figure 5(c) demonstrates monotonous decrease of the diffusion coefficient of methanol in acetonitrile in the entire range of densities and at all investigated temperatures. Fig. 15(d) is a typical example of anomalous density(pressure) dependence of the reorientation relaxation time. It is clear to see that it first decreases with density(pressure) and then starts to behave normally, i.e. increases with density(pressure). The order of decrease smoothly varies from about $40 \%$ at $T=258.15 \mathrm{~K}$ to about $3 \%$ at $T=298.15 \mathrm{~K}$. At $T=373.15 \mathrm{~K}$ anomaly is not observed, but the increase is very slow for the quite wide range of densities(pressures). In the case of acetonitrile in water, Fig. 5 (e), normal behavior of the reorientation relaxation time is observed almost entirely except for the lowest temperature, where $\tau$ has very shallow minimum. Finally, Fig. 5 (f) demonstrates monotonous increase of the reorientation relaxation time of methanol in acetonitrile in the entire range of densities and at all investigated temperatures. In such a way, it can be regarded as a typical example of normal density(pressure) behavior of $\tau$. Filled triangles and filled squares in Fig. [5 are results of MD simulation 9] at $T=298 \mathrm{~K}$ for methanol in water and acetonitrile in water, respectively, both at the infinite dilution. And asterisk symbols are experimental measurements [1] at $T=298.2 \mathrm{~K}$. One can see rather satisfactory correlation between the results of our computation, MD simulations and experiment, both qualitatively and quantitatively. It should be noted, however, that in terms of absolute values of $D$ and $\tau$ agreement with simulation and experimental data is poor.

Theoretical aspects of anomalous molecular mobility have been considered previously by the example of neat water [11]. Within the model employed, the authors proposed that the enhancement of the molecular mobility by compression can be related to the suppression of the number-density fluctuations in the low- $k$ region ( $k$ is the wave-number), rather than only the breakdown of the tetrahedral hydrogen-bonding network structure of water. The reasoning of this statement coherently follows from the examination of the behavior of memory functions 11 and is in harmony with the heuristic explanation of the picture 38. Let us imagine a molecule rotating in a polar media. The rotational motion will induce a relaxation process of surrounding molecules in order to make themselves aligned to the electric field produced by the new orientation of the molecule in concern. The energy dissipation associated with the relaxation process is an origin of the friction on the rotational motion, or the dielectric friction. The larger is the charge density fluctuation, the greater is the dielectric friction. When the liquid is compressed by an external pressure, the mechanism of electrostatic friction on the dielectric relaxation is mostly the same except that molecules in the solution are packed more tightly. Higher packing fraction prevents large number density fluctuations leading to smaller non-uniformity in the the polarization density and, as the result, smaller heterogeneity in the charge density. Then the electrostatic friction on dielectric relaxation is smaller and means that the dielectric relaxation time becomes shorter. Such an acceleration enhances the mobility of molecules in solution through the dielectric friction mechanism. Spherical shape of the molecule, or equivalently - spherical shape for the repulsive short-range interaction, favors anomalous molecular mobility. It is because the dominant contribution to the rotational and therefore dielectric friction is defined by the type of the interaction and its range, which explains the difference in the behavior of nearly spherical shape molecule of methanol and rod-like shape molecule of acetonitrile. For methanol the dominant contribution comes from the long-range Coulomb interaction, while for acetonitrile from the short-range repulsive one. As the result, methanol in water exhibits more substantial anomaly compared to acetonitrile in water. The anomalous behavior is attributed to the strong electrostatic interaction - the "hydrogen bond", among the solvent molecules and those between solute and solvent. The former causes the decrease in the dielectric relaxation time with pressure, while the latter induces the coupling between the dielectric mode of the solvent and the rotation of the solute. The anomaly is largely suppressed for acetonitrile in water due to enhanced significance of the repulsive core in the molecule. Finally, methanol in acetonitrile does not show any indication of the anomalous 

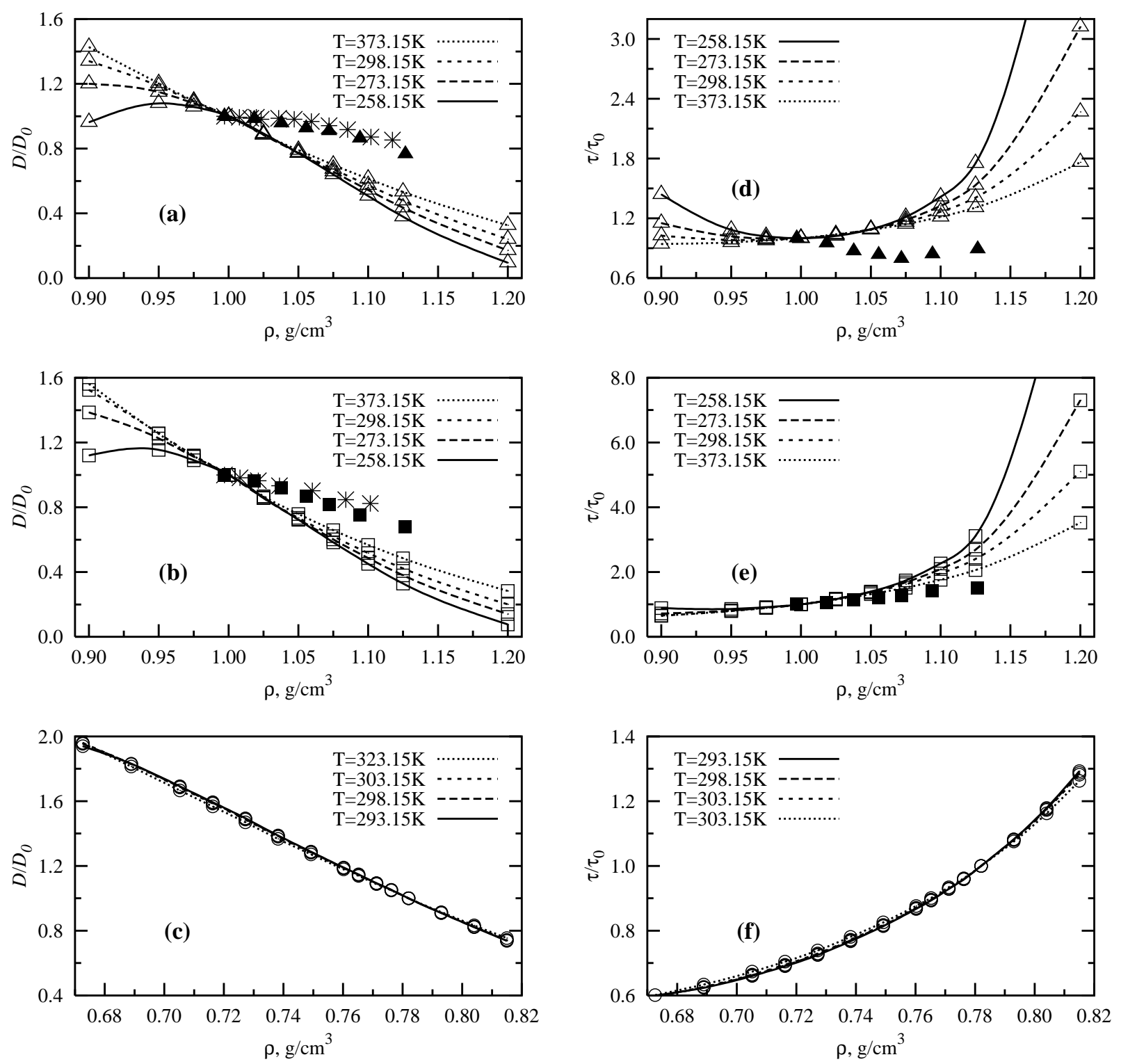

FIG. 5: Normalized translational diffusion coefficient $D / D_{0}$ and reorientation relaxation time $\tau / \tau_{0}$ for for methanol in water (a), (d); acetonitrile in water (b), (e); and for methaol in acetonitrile (c), (f), respectively. $\square, \triangle, 0-$ theory, $\mathbf{\square}, \boldsymbol{\Delta}-$ results of MD simulation [9] for $T=298 \mathrm{~K}, *$ - experimental data [1] for $T=298.2 \mathrm{~K}$, lines connecting open symbols are cubic splines for the eye-guide.

density dependence because of strong collisional friction on the collective reorientation of the solvent in this case, so that the dielectric relaxation becomes slower with pressure. Higher density or pressure just magnifies the effect of the repulsive core upon rotation and because of that the collisional friction.

\section{SUMMARY}

In present paper we have calculated the density dependence of the translational diffusion coefficients and rank-1 reorientation relaxation times for acetonitrile and methanol in water, and methanol in acetonitrile at various temperatures. Calculations have been performed using the site-site memory equation with the modecoupling approximation for memory kernels, and the DRISM/HNC for static properties. For simplicity of theoretical and computational procedures solutions have been considered in the limit of infinite dilution. Calculated quantities have been obtained to behave anomalously with density(pressure) for methanol in water. In particular, translational diffusion coefficient $D$ may increase for a while with density at low temperature (supercooled region), and reorientation relaxation time $\tau$ may decrease with density to form a minimum in the vicin- 
ity of ambient condition density for water in the quite wide range of temperatures. Similar computations for acetonitrile in water show tiny anomaly in the behavior of $D$ and $\tau$ only at the lowest (supercooled region) temperature, while for other regions there is no deviation from the usual behavior. And for methanol in acetonitrile there is no deviation in the entire region of investigated densities and temperatures. This picture is consistent with results of experimental observation and MD simulation, also quantitative agreement is not as good as qualitative. The physical origin of the anomalous density dependence of molecular mobility is interpreted in terms of two competing origins of friction, which interplay with each other as density increases: the collisional and dielectric frictions which, respectively, strengthen and weaken with increasing density. Presented results are first in scientific literature to realize anomalous molecular mobility of polar solute in water by means of the statistical mechanical theory.
[1] A. J. Easteal, L. A. Wolf, J. Phys. Chem. 89 (1985) 1066.

[2] C. Wakai, M. Nakahara, J. Chem. Phys. 100 (1994) 8347.

[3] C. Wakai, M. Nakahara, J. Chem. Phys. 106 (1997) 7512.

[4] C. Wakai, N. Matubayasi, M. Nakahara, J. Phys. Chem. A 103 (1999) 6685.

[5] K.R. Harris, P.J. Newitt, J. Chem. Eng. Data 42 (1997) 346.

[6] K. R. Harris, P. J. Newitt, J. Phys. Chem. B 102 (1998) 8874.

[7] K. R. Harris, P. J. Newitt, J. Phys. Chem. A 103 (1999) 6508.

[8] K. R. Harris, P. J. Newitt, J. Phys. Chem. B 103 (1999) 7015.

[9] S. Chowdhuri, A. Chandra, Chem. Phys. Lett. 373 (2003) 79.

[10] Molecular Theory of Solvation, ed. F. Hirata. Kluwer, Dordrecht, 2003.

[11] T. Yamaguchi, S.-H. Chong, F. Hirata, J. Chem. Phys. 119 (2003) 1021.

[12] In Ref. 10, Chapter 5: Dynamical Processes in Solution, by S.-H. Chong, p. 277-349.

[13] H. Mori, Progr. Theor. Phys. 33 (1965) 423.

[14] H. Mori, Progr. Theor. Phys. 34 (1965) 399.

[15] F. Hirata, P. J. Rossky, Chem. Phys. Lett. 83 (1981) 329.

[16] F. Hirata, B. M. Pettitt, P. J. Rossky, J. Chem. Phys. 77 (1982) 509.

[17] F. Hirata, P. J. Rossky, B. M. Pettitt, J. Chem. Phys. 78 (1983) 4133.

[18] T. Yamaguchi, S.-H. Chong, F. Hirata, J. Mol. Liquids 112 (2004) 117.

[19] J. Perkyns, M. B. Pettitt, Chem. Phys. Lett. 190 (1992) 626.

[20] J. Perkyns, M. B. Pettitt, J. Chem. Phys. 97 (1992) 7656.

[21] S.-H. Chong, F. Hirata, Phys. Rev. E 58 (1998) 7296.

[22] G. D. Harp, B. J. Berne, Phys. Rev. A 2 (1970) 975.

[23] J. P. Hansen, I. R. McDonald, Theory of Simple Liquids, 2nd ed. Academic Press, London, 1986.

[24] S.-H. Chong, F. Hirata, Phys. Rev. E 58 (1998) 6188.

[25] S.-H. Chong, W. Götze, Phys. Rev. E 65 (2002) 41503.

[26] T. Yamaguchi, F. Hirata, J. Chem. Phys. 117 (2002) 2216.

[27] H. J. J. C. Berendsen, J. R. Grigera, T. P. Straatsma, J. Phys. Chem. 91 (1987) 6269

[28] D. M. Edwards, P. A. Madden, I. R. McDonald, Mol. Phys. 51 (1984) 1141.

[29] W. L. Jorgensen, J. Phys. Chem. 90 (1986) 1276.

[30] W. Wagner, A. Pruß, J. Chem. Phys. Ref. Data 31 (2002) 387.

[31] Landolt-Börnstein Numerical Data and Functional Relationships in Science and Technology, New Series, ed. in chief K.H. Hellwege. Group IV: Macroscopic and Technical Properties of Matter. Vol. 4: High-Pressure Properties of Matter. G. Beggerow. Ed. Kl. Schäfer, Springer-Verlag, Berlin, 1980.

[32] H.-C. Ku, C.-H. Tu, J. Chem. Eng. Data 43 (1998) 465.

[33] P. S. Nikam, L. N. Shirsat, M. Hasan, J. Chem. Eng. Data 43 (1998) 732.

[34] Dielectric constants data from "Delta Construction Corporation", www.deltacnt.com.

[35] Dielectric constants data from "ASI Instruments Inc.", www.asiinstr.com.

[36] E. A. S. Cavell, H. G. Jerrard, B. A. W. Simmonds, J. A. Speed, J. Phys. Chem. 69 (1965) 3657.

[37] A. Kovalenko, S. Ten-no, F. Hirata, J. Comp. Chem. 20 (1999) 928.

[38] T. Yamaguchi, T. Matsuoka, S. Koda, J. Chem. Phys. 120 (2004) 7590. 